\title{
Heavy neutrino searches at future $Z$-factories
}

\author{
Jian-Nan Ding ${ }^{1, a}$, Qin Qin ${ }^{2,3, b}$, Fu-Sheng Yu ${ }^{1,4, c}$ \\ ${ }^{1}$ School of Nuclear Science and Technology, Lanzhou University, Lanzhou 730000, China \\ ${ }^{2}$ School of physics, Huazhong University of Science and Technology, Wuhan 430074, China \\ ${ }^{3}$ Theoretische Physik 1, Naturwissenschaftlich-Technische Fakultät Universität Siegen, Walter-Flex-Strasse 3, 57068 Siegen, Germany \\ ${ }^{4}$ Research Center for Hadron and CSR Physics, Lanzhou University and Institute of Modern Physics of CAS, Lanzhou 730000, China
}

Received: 15 March 2019 / Accepted: 5 September 2019 / Published online: 16 September 2019

(C) The Author(s) 2019

\begin{abstract}
We analyze the capacity of future $Z$-factories to search for heavy neutrinos with their mass from 10 to $85 \mathrm{GeV}$. The heavy neutrinos $N$ are considered to be produced via the process $e^{+} e^{-} \rightarrow Z \rightarrow v N$ and to decay into an electron or muon and two jets. By means of Monte Carlo simulation of such signal events and the Standard Model background events, we obtain the upper bounds on the cross sections $\sigma\left(e^{+} e^{-} \rightarrow v N \rightarrow v \ell j j\right)$ given by the $Z$-factories with integrated luminosities of $0.1,1$ and $10 \mathrm{ab}^{-1}$ if no signal events are observed. Under the assumption of a minimal extension of the Standard Model in the neutrino sector, we also present the corresponding constraints on the mixing parameters of the heavy neutrinos with the Standard Model leptons, and find they are improved by at least one order compared to current experimental constraints.
\end{abstract}

\section{Introduction}

In the Standard Model (SM), only left-handed neutrinos are introduced and no mechanism is responsible for the generation of neutrino mass. However, the observation of neutrino oscillations $[1,2]$ has given the evidence that neutrinos have tiny but non-zero mass, which may have opened a window towards the new dynamics beyond the SM. To explain the origin of neutrino mass and why they are much smaller than other fermion mass, different kinds of seesaw mechanisms [3-11] were proposed and work effectively as simple and straightforward methods. Among them, the Type-I Seesaw [3-6] is a quite natural extension of the SM by introducing gauge-singlet right-handed neutrinos without violating the SM gauge symmetries. Originally, it was proposed with the Majorana mass terms of the right-handed neutrinos at

\footnotetext{
a e-mail: dingjn13@1zu.edu.cn

be-mail: qin@physik.uni-siegen.de

c e-mail: yufsh@1zu.edu.cn
}

the scale of grand unified theories [12], which automatically lead to tiny neutrino mass. Later, it was found that a much lower Majorana mass scale, e.g. $\mathcal{O}(10) \mathrm{GeV}$, is also possible to explain the neutrino mass (see e.g. $[13,14]$ ), given small Dirac mass terms or some symmetry-protected cancellations in the neutrino mass matrix [15-19]. On the way to verify any of the Type-I Seesaw models, the most important evidence would be direct discoveries of heavy neutrinos. In this sense, the low-scale seesaw models are of special interest, because their particle spectra may contain heavy neutrinos with mass at $\mathcal{O}(10) \mathrm{GeV}$, within the reach of the colliders running now or in the near further. For example, extending the SM by three right-handed neutrinos with mass smaller than the electroweak scale [13,14], three heavy neutrinos beyond the SM spectrum are generated, one of which has a mass at $\mathrm{keV}$ scale as a dark-matter candidate and the other two at $\mathrm{GeV}$ to hundred $\mathrm{GeV}$ scale. Another interesting property of this model is that the mixing between the light neutrinos and the heavy neutrinos can be quite large. Therefore, heavy neutrinos in such a model have good opportunities to be detected by collider experiments. Once they are detected, it will also give us hints about leptonic CP violation as discussed in [20].

Experimentally, there have been direct searches for heavy neutrinos with $\mathcal{O}(10) \mathrm{GeV}$ mass by the DELPHI Collaboration [21]. In their searches, the heavy neutrinos were considered to be produced via the $e^{+} e^{-} \rightarrow Z \rightarrow v N$ process and decay into visible final states. Unfortunately, no signals were observed and thus only upper bounds on mixing parameters were given. There has also been a direct search by the CMS collaboration [22]. On the other hand, such heavy neutrinos also receive constraints from indirect searches like neutrinoless double-beta $(0 \nu 2 \beta)$ decays [2325], and can be explored in meson decays and $\tau$ decays [26]. Regarding future, there are several lepton colliders proposed by different communities, including the Circular Electron Positron Collider (CEPC) [27], the International Lin- 
ear Collider (ILC) [28], the FCC-ee (formerly known as the TLEP) [29] and the Super $Z$ Factory. While the main target of most of these colliders is precision study of the Higgs boson properties, they will also be capable of searching for some new particles, new dynamics and even of studies of quantum chromodynamics and hadrons (see e.g. [30-34]). Most importantly here, they will be ideal facilities to search for heavy neutrinos. Such abilities of the CEPC with $\sqrt{s}=$ 240-250 GeV and of the ILC with $\sqrt{s}=1 \mathrm{TeV}$ have been investigated by [35,36]. One can refer to e.g. [37-43] for collider searches for neutrinos with higher masses.

In this work, we will focus on searching for heavy neutrinos $N$ with $\mathcal{O}(10) \mathrm{GeV}$ mass at future $Z$-factories with integrated luminosities of $0.1,1$ and $10 \mathrm{ab}^{-1}$. Similar to DELPHI, we will also consider the signal production process $e^{+} e^{-} \rightarrow Z \rightarrow v N$ of heavy neutrinos, and reconstruct the heavy neutrinos by their visible decaying final states each containing one charged lepton and two quark jets. The background mainly originates from the SM processes $e^{+} e^{-} \rightarrow j j j j, \tau^{+} \tau^{-}$and $b \bar{b}$. Compared to a Higgs factory such as the CEPC with $\sqrt{s}=240-250 \mathrm{GeV}$, we find that future $Z$-factories are much more sensitive to heavy neutrinos with mass below $80 \mathrm{GeV}$, because the $N$ production cross sections at the $Z$-mass pole is typically higher than those at $240-250 \mathrm{GeV}$ by orders. We have noticed that employing the technique of displaced-vertex detection [44,45], a search for heavy neutrinos at a $Z$-factory is almost free of background and can thus set even more stringent constraints to the mixing parameters between the heavy neutrinos and the SM leptons than a normal search. However, the prerequisite of the displaced-vertex technique depends on a strong assumption that the studied heavy neutrinos have lifetimes long enough to fly a detectable distance before decaying, which is not true for many models. For example, if a heavy neutrino has decay channels with large decay widths, then it will have a very short lifetime so that its decay vertex is "not displaced". Heavy neutrinos in a model with a Majoron $J[46,47]$, which was introduced to generate the Majorana scale in an ultraviolet-complete way, have such a feature. Typically, they can efficiently decay into the light Majoron via the invisible channels $N \rightarrow v J$ and thus have short lifetimes. Therefore, while the constraints given by $[44,45]$ do not apply to such heavy neutrinos, those given in this work are still valid. In other words, the results of our work without making use of the displaced-vertex technique are more model independent than $[44,45]$.

The rest of the paper is orgnized as follows. In Sect. 2, we will introduce the general setup of the new-physics scenario that we consider. The simulation of the production and decay processes of the heavy neutrinos and the corresponding background events at $Z$-factories will be described, and the event selection conditions will be explained in Sect. 3 . We will present the results in Sect. 4 and conclude by Sect. 5 .

\section{General setup of the scenario}

In this work, we consider a scenario generating the tiny neutrino mass via the Type-I seesaw mechanism but with a low Majorana mass scale (see e.g. $[13,14]$ ). It introduces $n$ righthanded neutrinos $R_{j}(j=1, \ldots, n)$, which are singlets of the SM gauge group SU(2), and their kinetic terms, mass terms and interaction terms with the SM fields are given by

$$
\begin{aligned}
\mathcal{L} \ni & \frac{1}{2} \sum_{j} \bar{R}_{j} i \not \partial R_{j}-\sum_{i, j} y_{i j} \bar{L}_{i} \widetilde{H} R_{j}-\frac{1}{2} \sum_{j} \bar{R}_{j}^{c} M_{R} R_{j} \\
& + \text { h.c. }
\end{aligned}
$$

where $\widetilde{H}=i \tau_{2} H^{*}$, the lepton $\mathrm{SU}(2)_{L}$ doublet $L=$ $\left(v_{\ell L}, \ell_{L}\right)^{\mathrm{T}}$ with $\ell=e, \mu, \tau, y$ is the $3 \times n$ Yukawa coupling matrix and the $n \times n$ Majorana mass matrix $M_{R}$ is generated by some high-scale dynamics. After the spontaneous symmetry breaking of the Higgs field, we can diagonalise the neutrino mass matrix and obtain the $3+n$ mass-eigenstate neutrinos, including 3 light neutrinos $v_{i}$ and $n$ heavy neutri$\operatorname{nos} N_{j} .{ }^{1}$ Now, a flavor eigenstate is a superposition of the mass eigenstates, as

$\nu_{\ell}=\sum_{i=1}^{3} U_{\ell i} v_{i}+\sum_{j=4}^{3+n} V_{\ell j} N_{j}, \quad$ with $\ell=e, \mu, \tau$,

and thus the neutrino-relevant weak interaction terms are given by

$$
\begin{aligned}
\mathcal{L} \ni- & \frac{g}{2 \cos \theta_{W}} Z_{\mu} \sum_{\ell}\left(\sum_{i=1}^{3} U_{\ell i}^{*} \bar{\nu}_{i}+\sum_{j=4}^{3+n} V_{\ell j}^{*} \bar{N}_{j}\right) \\
& \times \gamma^{\mu} P_{L}\left(\sum_{i^{\prime}=1}^{3} U_{\ell i^{\prime}} \nu_{i^{\prime}}+\sum_{j^{\prime}=4}^{3+n} V_{\ell j^{\prime}} N_{j^{\prime}}\right) \\
& -\left[\frac { g } { \sqrt { 2 } } W _ { \mu } ^ { + } \sum _ { \ell } \left(\sum_{i=1}^{3} U_{\ell i}^{*} \bar{\nu}_{i} \gamma^{\mu} P_{L} \ell\right.\right. \\
& \left.\left.+\sum_{j=4}^{3+n} V_{\ell j}^{*} \bar{N}_{j} \gamma^{\mu} P_{L} \ell\right)+ \text { h.c. }\right] .
\end{aligned}
$$

It has been proved by [48] that the non-diagonal weak currents here do not vanish because $\nu_{\ell}$ and $R_{j}$ are different representations of the weak $\mathrm{SU}(2)$ group.

From the above interaction terms (3), we read out that a heavy neutrino (with the mass smaller than the $Z$ boson mass) can be produced associated with light neutrinos via

\footnotetext{
${ }^{1}$ For clarification, here $N_{j}$ is not a Majorana neutrino by itself, but the left-handed component of a Majorana neutrino. It corresponds to $N_{j}^{c}$ in [26].
} 


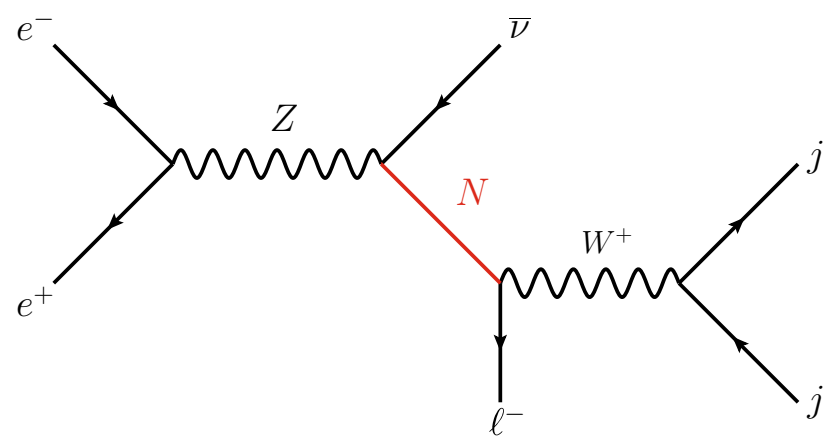

Fig. 1 The Feynman diagram of the signal with $\ell=e$ or $\mu$

$e^{+} e^{-} \rightarrow Z \rightarrow \bar{v} N(v \bar{N})$, which is actually the dominant production process. ${ }^{2}$ We also find that the produced heavy neutrino can decay weakly into one charged lepton and one on-shell or off-shell $W$ boson up to its mass, $N \rightarrow \ell^{-} W^{+(*)}$. Here, to reconstruct the heavy neutrinos, we choose the signal events with the decaying products including one charged lepton and two jets, all of which can be collected by detectors, as displayed in Fig. 1. The kinematics requires that the mass of the heavy neutrinos are smaller than the $Z$ boson mass.

To get a hint of the potential of a $Z$-factory in searching for such heavy neutrino signals, we compare the performances of a $Z$-factory and a Higgs factory with the electron-positron collision energy at $M_{Z}=91.2 \mathrm{GeV}$ and $240 \mathrm{GeV}$, respectively. Under the assumption of the new dynamics coming only from the terms in (1), the dependence of the signal production cross sections $\sigma\left(e^{+} e^{-} \rightarrow \nu \ell j j\right)^{3}$ on the corresponding mixing parameters $\left|V_{\ell N}\right|^{2}$ is obtained and shown in Fig. 2, with $\ell=e$ and $\mu$. In either the $\ell=e$ or $\ell=\mu$ case, we consider only the heavy neutrino mixing with the electron or the muon sector, such that the production cross sections only depend on the mixing parameters $\left|V_{e N}\right|^{2}$ or $\left|V_{\mu N}\right|^{2}$. The results are calculated by MadGraph [49] with the new dynamics implemented via FeynRules [50-52]. We find that if the heavy neutrino mass $10 \mathrm{GeV}<M_{N}<80 \mathrm{GeV}$, the signal cross sections at a $Z$-factory exceed $10^{3}\left|V_{\ell N}\right|^{2} \mathrm{fb}$, while the cross sections at a Higgs factory are smaller by 1 to 2 orders in the $\ell=e$ case and by 3 to 4 orders in the $\ell=\mu$ case. The searching for heavy neutrinos with the mass between $10 \mathrm{GeV}$ and $85 \mathrm{GeV}$ at a $Z$-factory is obviously better than that at a Higgs factory with the center-of-mass energy of $240 \mathrm{GeV}$ and a similar luminosity.

\footnotetext{
${ }^{2}$ Even when $Z \rightarrow N \bar{N}$ is kinematically allowed, the cross section is doubly suppressed by the tiny mixing matrix elements $\left|V_{\ell j}\right|^{2}$, which receive stringent constraints from previous experiments such as the DELPHI [21].

${ }^{3}$ By $\nu \ell$ we always sum over all possible leptons and their antiparticles, including both the lepton-number-conserving final states like $\bar{v} \ell^{-}$and the lepton-number-breaking ones like $\nu \ell^{-}$.
}

Next, we emphasize that even we switch on the mixing between heavy neutrinos and all the other lepton sectors, the signal cross sections $\sigma\left(e^{+} e^{-} \rightarrow v N \rightarrow v \ell j j\right)$ at a $Z$ factory still basically only depend on the one corresponding mixing parameter $\left|V_{\ell N}\right|^{2}$. The reason is as follows. The cross section $\sigma\left(e^{+} e^{-} \rightarrow v N_{k}\right)$ summing up three light neutrinos and their anti-particles is proportional to the mixing parameter $\sum_{i=1}^{3}\left|\left(U^{\dagger} V\right)_{i k}\right|^{2}$, and under the limit that the matrix $U$ is almost unitary, we have $\sum_{i=1}^{3}\left|\left(U^{\dagger} V\right)_{i k}\right|^{2} \approx$ $\sum_{\ell}\left|V_{\ell k}\right|^{2}$. On the other hand, safely neglecting the difference between the charged lepton mass in $N_{k}$ decays, we have that $\mathcal{B}\left(N_{k} \rightarrow \ell j j\right) \propto\left|V_{\ell k}\right|^{2} / \sum_{\ell^{\prime}}\left|V_{\ell^{\prime} k}\right|^{2}$. Therefore, the two $\sum_{\ell}\left|V_{\ell j}\right|^{2}$ factors get cancelled when we multiply $\sigma\left(e^{+} e^{-} \rightarrow v N_{k}\right)$ by $\mathcal{B}\left(N_{k} \rightarrow \ell j j\right)$, and the relation $\sigma\left(e^{+} e^{-} \rightarrow \nu N_{k} \rightarrow \nu \ell j j\right) \propto\left|V_{\ell k}\right|^{2}$ is obtained. Here, we emphasize that unless specially noted, our analysis in this work will not depend on the assumption of this paragraph and the previous paragraph, which means that the validity of the results of this work is not limited to the specific model extending the SM with only the (1) terms.

The small peaks appearing where $M_{N}$ is slightly above the $W$ boson mass are due to the opening of $N$ decaying into an on-shell $W$ boson. The decline of the cross sections at the mass close to $90 \mathrm{GeV}$ results from the suppression of the phase space.

\section{Event simulation and selection}

For event simulation, we use MadGraph [49] as the event generator for both the SM background and the signal with the new dynamics implemented via FeynRules [50-52] as mentioned previously. After that, Pythia8 [53] and Delphes [54] are used for further hadronization and fast detector simulation, respectively. In the detector simulation, the eekt algorithm and exclusive search have been chosen to construct jets, which, compared to the default antikt algorithm, is more efficient for a lepton collider and mitigates energy peak drifts of jets.

The signal events are produced from the processes $e^{+} e^{-} \rightarrow v N \rightarrow v \ell j j$, and in this work we consider the charged lepton $\ell$ to be an electron or a muon. Therefore, the final states are forced into three jets (one leptonic jet and two hadronic jets for example) with the the eekt algorithm. In both the electron and muon cases, the main background comes from the $e^{+} e^{-} \rightarrow j j j j, b \bar{b}$ and $\tau^{+} \tau^{-}$processes. In one $j j j j$ event, if one jet is too soft or collinear to the beam and is thus not detected but identified as missing energy, and simultaneously another jet is misidentified as an electron or muon, such an event may mimic a signal event. In this work, we assume that the misidentification rates of jets as electrons and muons are about $10^{-4}$. As for the $b \bar{b}$ and $\tau^{+} \tau^{-}$events, 

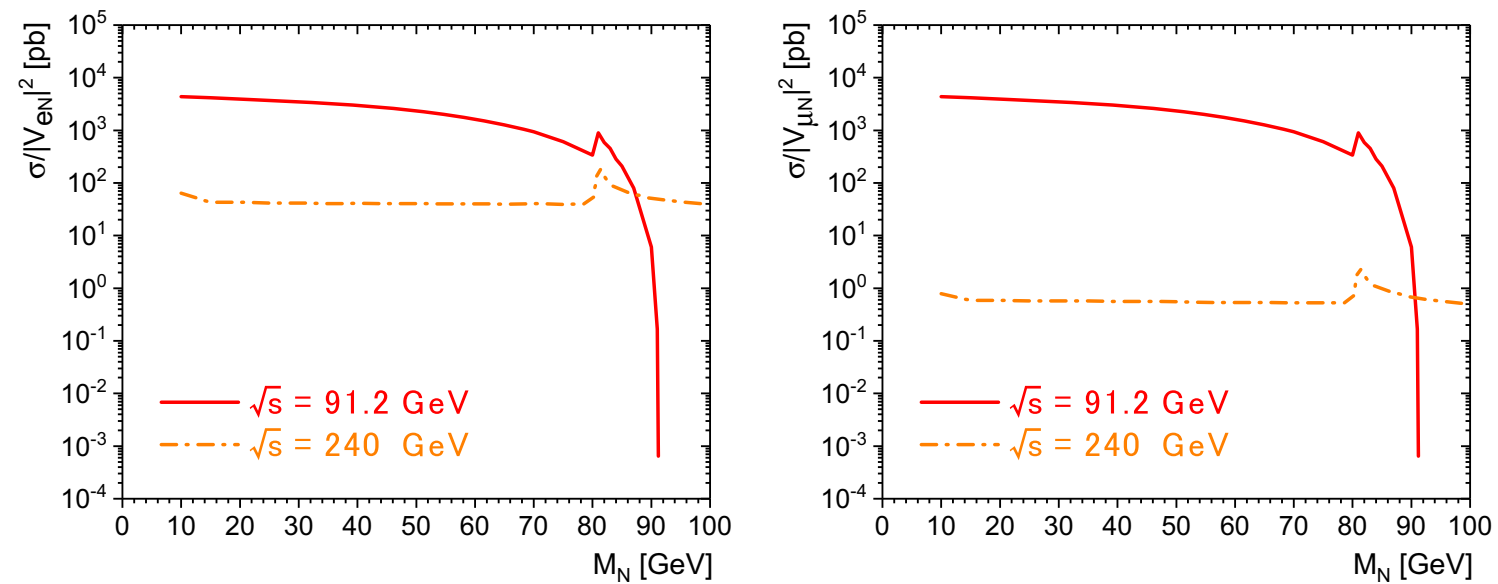

Fig. 2 The production cross sections of $e^{+} e^{-} \rightarrow v N \rightarrow v e j j$ (left) and $e^{+} e^{-} \rightarrow v N \rightarrow v \mu j j$ (right) at a Z-factory (red) and a Higgs factory (orange) with the heavy neutrino mass $M_{N}$ varying from 10 to $100 \mathrm{GeV}$

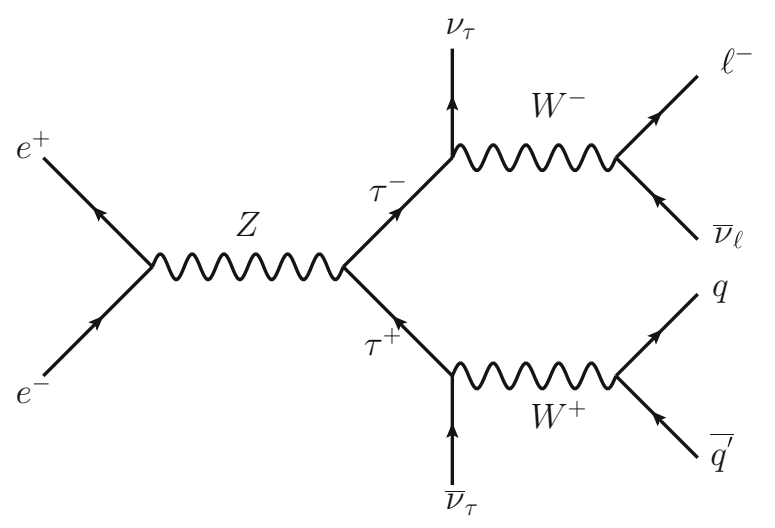

Fig. 3 Sample Feynman diagrams of the background

the fermion pairs further decay into final states containing one charged lepton, two jets and missing energy, $\mathbb{E} \ell j j$, as shown in Fig. 3. It should be pointed out that, although the decay products of a $\tau$ lepton are usually recognized as one single jet, in the eekt-exclusive algorithm it is possible that the products are reconstructed into two jets. This is due to that this algorithm automatically cluster nearest objects step by step until the final state of the event is reconstructed to exactly 3 jets. As for the other $q \bar{q}$ events with $q$ being a lighter quark, production at the $Z$-mass pole makes them highly boosted such that it is more difficult for the decay products of $q$ or $\bar{q}$ to form two open jets. Therefore, it is much more difficult for a lighter quark pair $q \bar{q}$ to mimic a signal event than a $b \bar{b}$ pair.

In the following, we will discuss how we choose the event selection conditions to suppress the background and increase the signal significance. The $j j j j$ events that mimic signal events typically have missing energy with small transverse momenta $\mathbb{E}_{T}$. Therefore, a cut requiring a least $\mathbb{E}_{T}$ can efficiently suppress such background.

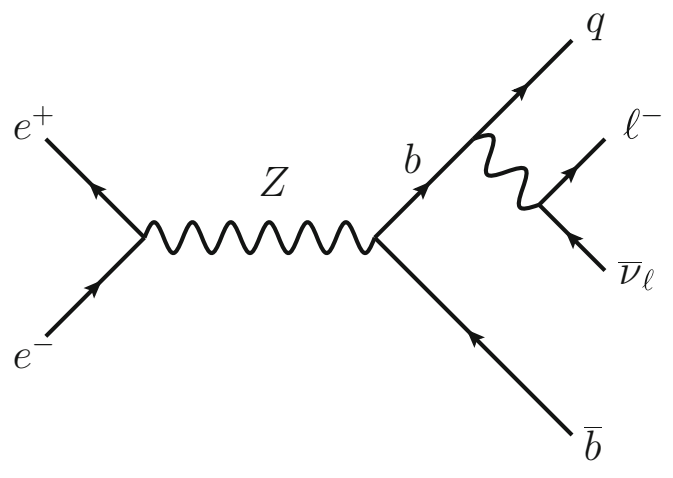

For the $\tau \tau$ background as shown in the left panel of Fig. 3, the two $\tau$ leptons in an event fly back-to-back with a large boost. Therefore, the charged lepton and the total missing energy in the final state are almost collinear or reverse to each other, and the angle between the two jets decaying from the same $\tau$ are very small. These inspire some effective cuts on the angular distances $\Delta R_{j j}$ and $\Delta R_{\mathbb{E} \ell}$, where $\Delta R=\sqrt{\Delta \eta^{2}+\Delta \phi^{2}}$ with $\Delta \eta$ and $\Delta \phi$ being the differences between the pseudorapidities and the azimuthal angles of the two involved objects, respectively.

In a $b \bar{b}$ background event as shown in the right panel of Fig. 3, the neutrino, the charged lepton and one of the two jets decay from the same bottom quark with a large boost, so not only the angular distance between the charged lepton and the neutrino but also the angular distance between the jet and the neutrino are small. Therefore, we set cuts on $\Delta R_{E \ell}$ and $\Delta R_{\mathbb{E} j}$ to reduce the background from the $b \bar{b}$ process.

Considering the signal process $e^{+} e^{-} \rightarrow v N$, the energy of the resolved light neutrino is fixed owing to the momentumenergy conservation, as 
$E_{v}=\frac{M_{Z}^{2}-M_{N}^{2}}{2 M_{Z}}$,

which can also provide an effective cut to suppress the background. In practice, the missing energy in any event is reconstructed by summing the 3-momenta of the visible objects, $\mathbb{E}=\left|\Sigma_{i} \mathbf{p}_{i}\right|$. If an event only contains one neutrino, $\mathbb{E}$ is honestly the neutrino energy $E_{v}$ and the reconstructed total energy $E_{\mathrm{rec}}=\mathbb{E}+\Sigma_{i} E_{i}$ reproduces the collision energy, about $91.2 \mathrm{GeV}$. Otherwise, if an event contains more than one undetected object (only one undetected massive object also has such a property), the reconstructed total energy $E_{\text {rec }}$ may deviate from the collision energy. Therefore, we also use $E_{\text {rec }}$ cuts to suppress the corresponding background.

Inspired by the above considerations and some practical tests, we choose the following event selection conditions for the three categories depending on different heavy neutrino mass, the small-mass range $\left(M_{N}<65 \mathrm{GeV}\right)$, the middlemass range $\left(65 \mathrm{GeV}<M_{N}<80 \mathrm{GeV}\right)$ and the large-mass range $\left(80 \mathrm{GeV}<M_{N}<85 \mathrm{GeV}\right)$.

- The event selection conditions for the small-mass range $\left(M_{N}<65 \mathrm{GeV}\right)$ :

- $P_{T}^{j}>5 \mathrm{GeV},\left|\eta_{j}\right|<2, \Delta R_{j j}>0.1$, btag $<0.8$, TauTag $=0$, BTag $=0$;

- $P_{T}^{\ell}>3 \mathrm{GeV},\left|\eta_{\ell}\right|<1$;

- $\mathbb{E}_{T}>20 \mathrm{GeV},\left|E_{\text {rec }}-M_{Z}\right|<10 \mathrm{GeV}, \mid M_{\ell j j}-$ $M_{N} \mid<\Gamma_{1 / 2}^{M}$;

$-1.0<\Delta R_{\mathbb{E} j}<5.5,1.5<\Delta R_{\mathbb{E} \ell}<5.0 ;$

- the event selection conditions for the middle-mass range $\left(65<M_{N}<80 \mathrm{GeV}\right)$ :

- $P_{T}^{j}>5 \mathrm{GeV},\left|\eta_{j}\right|<2, \Delta R_{j j}>0.4$, btag $<0.8$, TauTag $=0$, BTag $=0$;

- $P_{T}^{\ell}>3 \mathrm{GeV},\left|\eta_{\ell}\right|<1$;

- $\mathbb{E}_{T}>10 \mathrm{GeV},\left|E_{\mathrm{rec}}-M_{Z}\right|<10 \mathrm{GeV},\left|\mathbb{E}-\mathbb{E}_{0}\right|<$ $\Gamma_{1 / 2}^{E},\left|M_{\ell j j}-M_{N}\right|<\Gamma_{1 / 2}^{M}$;

$-1.0<\Delta R_{\mathbb{E} j}<5.5,1.5<\Delta R_{\mathbb{E} \ell}<5.0$;

- the event selection conditions for the large-mass range $\left(80<M_{N}<85 \mathrm{GeV}\right)$ :

- $P_{T}^{j}>10 \mathrm{GeV},\left|\eta_{j}\right|<2, \Delta R_{j j}>0.4, M_{j j}>$ $55 \mathrm{GeV}$, btag $<0.8$, TauTag $=0$, BTag $=0$;

- $P_{T}^{\ell}>3 \mathrm{GeV},\left|\eta_{\ell}\right|<1$;

- $\mathbb{E}_{T}>5 \mathrm{GeV},\left|E_{\mathrm{rec}}-M_{Z}\right|<10 \mathrm{GeV},\left|\boldsymbol{E}-\mathbb{E}_{0}\right|<$ $\Gamma_{1 / 2}^{E},\left|M_{\ell j j}-M_{N}\right|<\Gamma_{1 / 2}^{M}$;

$-1.5<\Delta R_{\mathbb{E} j}<5.5,1.5<\Delta R_{\mathbb{E} \ell}<5.0$.

In general, we set cuts on the transverse momenta $P_{T}$ and the pseudorapidities $|\eta|$ of the charged lepton and the jets, the transverse missing energy $\mathbb{E}_{T}$, the invariant mass of the two jets $M_{j j}$, the reconstructed total energy $E_{\mathrm{rec}}$, the angular distances between the two jets $\Delta R_{j j}$, between each jet and the missing energy $\Delta R_{\mathbb{E} j}$ and between the missing energy and the charged lepton $\Delta R_{\mathbb{E} \ell}$. Note that the $P_{T}^{j}$ cut can not be chosen to be too low, because the Monte Carlo simulation does not fully model physics at very low scales. It should be totally safe if we set a cut of $P_{T}^{j}>10 \mathrm{GeV}$, which we accept for the large- $M_{N}$ range. We have also attempted such a $P_{T}^{j}$ cut for the other two mass ranges, but find that in such a case the signal reconstruction efficiency is extremely suppressed. Therefore, the result with a $P_{T}^{j}>10 \mathrm{GeV}$ cut in the small- and middle- $M_{N}$ ranges would not honestly reflect the real sensitivity of a $Z$-factory, and we choose $P_{T}^{j}>5 \mathrm{GeV}$ instead. In addition, from the signal simulation we get the central value of the detected missing energies $\mathbb{E}_{0}$ and the halfheight width $\Gamma_{1 / 2}^{\mathscr{E}}$ of the distribution, and we require that the missing energy $\mathbb{E}$ in each event does not lie beyond the region $\mathbb{E}_{0} \pm \Gamma_{1 / 2}^{\mathbb{E}}$. A sizable width $\Gamma_{1 / 2}^{\mathbb{E}}$ is formed mainly because the energy resolution for jets and leptons is not perfect. We also require that the invariant mass of the lepton and the dijet $M_{\ell j j}$ should lie in the region $M_{N} \pm \Gamma_{1 / 2}^{M}$, where $\Gamma_{1 / 2}^{M}$ is the half-width of the $M_{N}$ spectrum of the reconstructed signal events. To further suppress the background, we also use the btag, BTag and TauTag, which gives information of the probability of a jet being a $b$-jet, whether or not a jet has been tagged as containing a heavy quark and whether or not a jet has been tagged as a tau, respectively.

After the event selection, we find that the $j j j j$ events dominate the background for all the three $M_{N}$ ranges. Besides, while the $b \bar{b}$ and $\tau \tau$ contributions to the background are considerable, the other contributions like $c \bar{c}, j j \ell \ell$ and $\ell \ell \ell \ell$ are negligible.

\section{Results and analysis}

In this section, based on the simulation of the signal and background events, we present the capacity of $Z$-factories to search for heavy neutrinos with three benchmark integrated luminosities $\mathcal{L}, 0.1 \mathrm{ab}^{-1}, 1 \mathrm{ab}^{-1}$ and $10 \mathrm{ab}^{-1}$. In practice, we estimate the expected upper bounds on the cross sections $\sigma\left(e^{+} e^{-} \rightarrow \nu N \rightarrow v \ell j j\right)$ at $95 \%$ confidence level (CL), which can be approximately obtained by solving the equation

$s=\frac{N_{s}}{\sqrt{N_{B}+N_{s}}}=\frac{N_{s 0} \times\left(\sigma / \sigma_{0}\right)}{\sqrt{N_{B 0}+N_{s 0} \times\left(\sigma / \sigma_{0}\right)}} \sqrt{\frac{\mathcal{L}}{\mathcal{L}_{0}}}$,

with $s \approx 1.7$. This equation is understood as follows. For convenience, we only perform the simulation once for each heavy neutrino mass, with a reference setup of a specific luminosity $\mathcal{L}_{0}$ and a specific cross section of the signal $\sigma_{0}$, which brings $N_{B 0}$ detected background events and $N_{s 0}$ detected signal events. Then, based on the simulation, other 

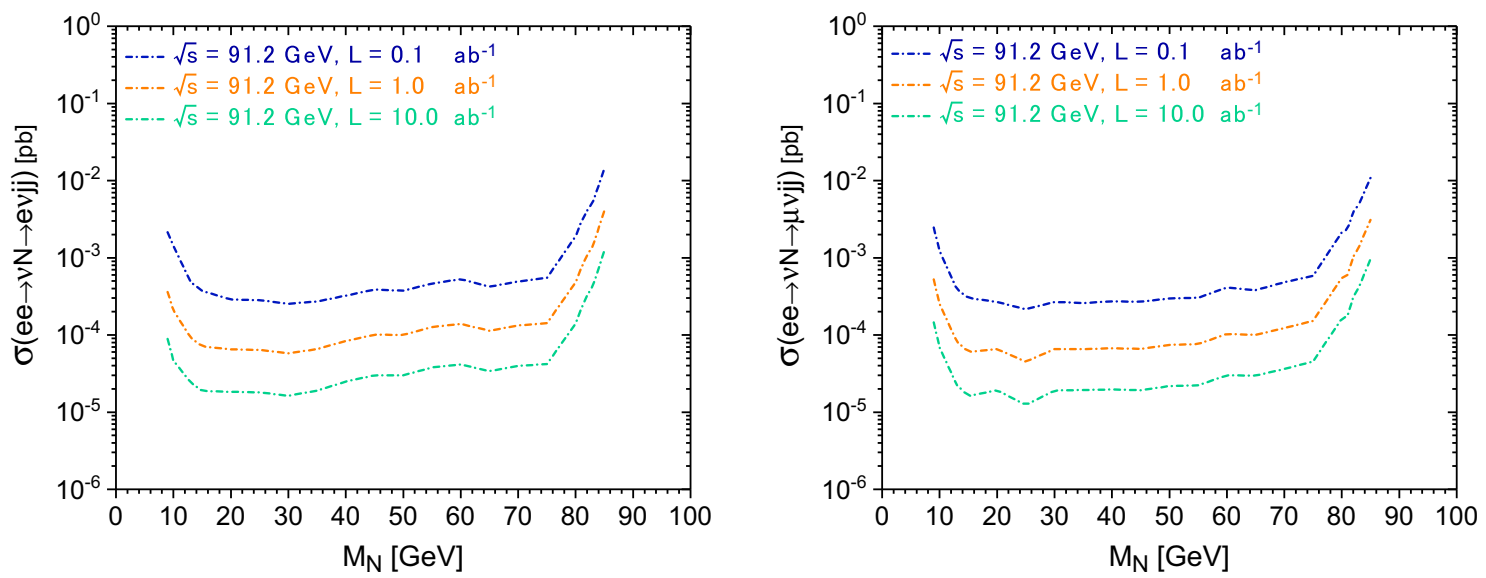

Fig. 4 The upper bounds on $\sigma\left(e^{+} e^{-} \rightarrow v N \rightarrow v e j j\right)$ (left) and $\sigma\left(e^{+} e^{-} \rightarrow v N \rightarrow v \mu j j\right)$ (right) by Z-factories at 95\% CL. The blue, orange and cyan curves correspond to integrated luminosities of $0.1 \mathrm{ab}^{-1}, 1.0 \mathrm{ab}^{-1}$ and $10 \mathrm{ab}^{-1}$, respectively. See text for details

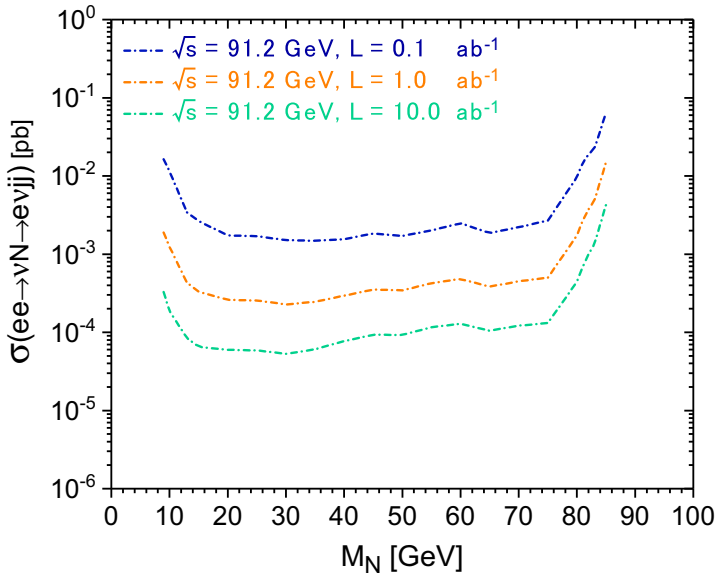

Fig. 5 The smallest $\sigma\left(e^{+} e^{-} \rightarrow v N \rightarrow v e j j\right)$ (left) and $\sigma\left(e^{+} e^{-} \rightarrow v N \rightarrow v \mu j j\right)$ (right) required to have heavy neutrinos discovered at $Z$-factories. The blue, orange and cyan curves correspond to

cases are obtained by scaling the luminosity and the cross section, as expressed in the second equality of the formula (5).

The main results of this work, the upper bounds on $\sigma\left(e^{+} e^{-} \rightarrow v N \rightarrow v e j j\right)$ and $\sigma\left(e^{+} e^{-} \rightarrow v N \rightarrow v \mu j j\right)$ at 95\% CL given by future $Z$-factories, are shown in Fig. 4 , with the heavy neutrino mass varying from 10 to $85 \mathrm{GeV}$. The curves for the integrated luminosities of $0.1 \mathrm{ab}^{-1}, 1 \mathrm{ab}^{-1}$ and $10 \mathrm{ab}^{-1}$ are presented. We find that for most of the mass range, i.e. $15 \mathrm{GeV}<M_{N}<75 \mathrm{GeV}$, the upper bounds on the production cross sections are around a few $10^{-4}$ $\mathrm{pb}$ to $10^{-5} \mathrm{pb}$ in both the electron and muon cases, with the integrated luminosities varying from $0.1 \mathrm{ab}^{-1}, 1 \mathrm{ab}^{-1}$ and $10 \mathrm{ab}^{-1}$. Also, we assume that a significance $s$ larger than 5 in (5) indicates discovery of heavy neutrinos, and show the corresponding smallest discovery cross sections $\sigma\left(e^{+} e^{-} \rightarrow v N \rightarrow v e j j\right)$ and $\sigma\left(e^{+} e^{-} \rightarrow v N \rightarrow v \mu j j\right)$ in Fig. 5.

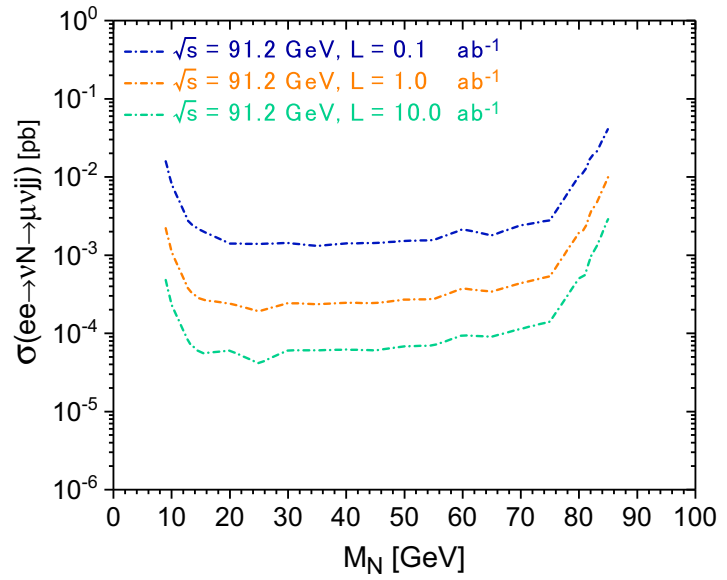

integrated luminosities of $0.1 \mathrm{ab}^{-1}, 1.0 \mathrm{ab}^{-1}$ and $10 \mathrm{ab}^{-1}$, respectively. See text for details

In principle, the measurement of the total width of the $Z$ boson $\Gamma_{Z}=2.4952 \pm 0.0023 \mathrm{GeV}$ [55] also sets constraints to the $e^{+} e^{-} \rightarrow Z \rightarrow v N$ cross sections, but we find that heavy neutrinos with their signal cross sections below $Z$-factory bounds in Fig. 4 automatically satisfy the $\Gamma_{Z}$ constraints. For example, we consider a heavy neutrino with $\sigma\left(e^{+} e^{-} \rightarrow v N \rightarrow v \ell j j\right)$ of $\mathcal{O}\left(10^{-2}\right) \mathrm{pb}$, which is the largest allowed cross section if no signals are observed at a $\mathcal{L}=0.1 \mathrm{ab}^{-1}$ machine. It corresponds to an additional contribution $\Gamma(Z \rightarrow v N \rightarrow v \ell j j)$ of $\mathcal{O}\left(10^{-7}\right) \mathrm{GeV}$ to the $Z$ decay width. If the branching ratio $\mathcal{B}(N \rightarrow \ell j j)$ is of $\mathcal{O}(10 \%)$, then the existence of such a heavy neutrino will enlarge the total $Z$ decay width by $\Gamma(Z \rightarrow v N)$ of $\mathcal{O}\left(10^{-6}\right) \mathrm{GeV}$, which is smaller than the uncertainty of the data $\Gamma_{Z}=2.4952 \pm 0.0023 \mathrm{GeV}$ by three orders. In other words, the constraint from the measurement of $\Gamma_{Z}$ is not comparable with the constraints given by this work. 


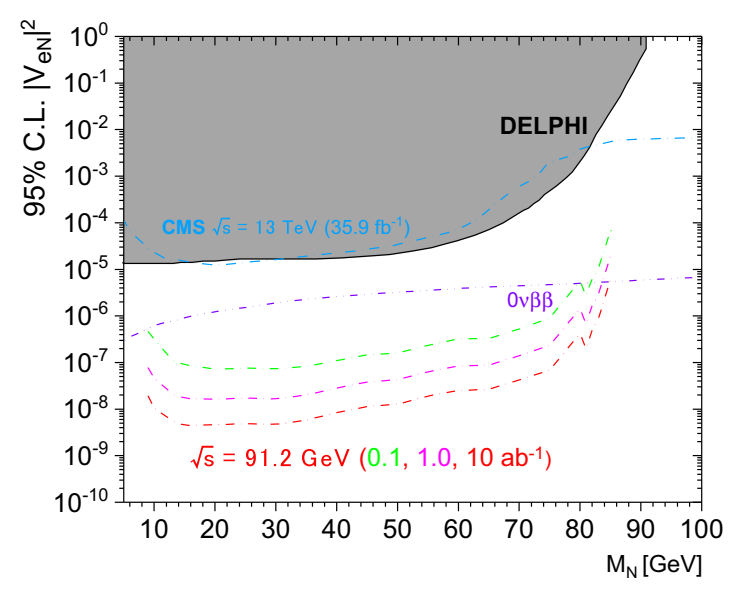

Fig. 6 The upper bounds on the mixing parameters $\left|V_{e N}\right|^{2}$ (left) and $\left|V_{\mu N}\right|^{2}$ (right) given by $Z$-factories at 95\% CL, compared to the upper bounds given by DELPHI [21] and CMS [22], the $0 \nu 2 \beta$ decay exper-

To have a direct comparison with previous experimental constraints and some relevant future ones, we consider the case in which (1) is the only source of new dynamics beyond the SM and give the $Z$-factory constraints on the mixing parameters $\left|V_{e N}\right|^{2}$ and $\left|V_{\mu N}\right|^{2}$ in Fig. 6. ${ }^{4}$ This is achievable because the cross sections are proportional to the corresponding mixing parameters, $\sigma\left(e^{+} e^{-} \rightarrow v N \rightarrow v \ell j j\right) \propto\left|V_{\ell N}\right|^{2}$, as analysed in the last paragraph of Sect. 2. For both $\left|V_{e N}\right|^{2}$ and $\left|V_{\mu N}\right|^{2}$, we find a large improvement compared to DELPHI [21] and CMS [22], the upper bounds being decreased typically by two orders of magnitude even with the lowest luminosity setup. For $\left|V_{e N}\right|^{2}$, the given upper bounds by the considered $Z$-factories are lower than that given by the $0 \nu 2 \beta$ decay experiments [23-25] by at least one order of magnitude in most of the mass range of the heavy neutrino. While for $\left|V_{\mu N}\right|^{2}$, the upper bounds given by the $Z$-factories are at least two orders of magnitude lower than that given by the CEPC as a Higgs factory [36] when $M_{N}<70 \mathrm{GeV}$. One may worry that a heavy neutrino with mixing parameters as small as such bounds will be so stable that the detection of its decay is always out of reach by detectors. If this is true, the bounds given in Fig. 6 will not be valid, since all our analyses are based on the assumption that the signal events can be detected within detectors once they happen. To clarify that this will not be a problem, we estimate how far a $10 \mathrm{GeV}$ heavy neutrino can typically fly before its decay, and the flying distances of the other heavier neutrinos are always shorter given the same relevant mixing parameters. Considering a detector having a diameter of $\mathcal{O}(1)$ meter, we find that as long as the mixing parameter $\sum_{\ell}\left|V_{\ell N}\right|^{2}$ is larger than $\mathcal{O}\left(10^{-9}\right)$, the $10 \mathrm{GeV}$

\footnotetext{
${ }^{4}$ We are aware that in such a case $Z$-factories are able to give much more stringent constraints on these mixing parameters than our constraints by making use of displaced vertex information $[44,45]$.
}

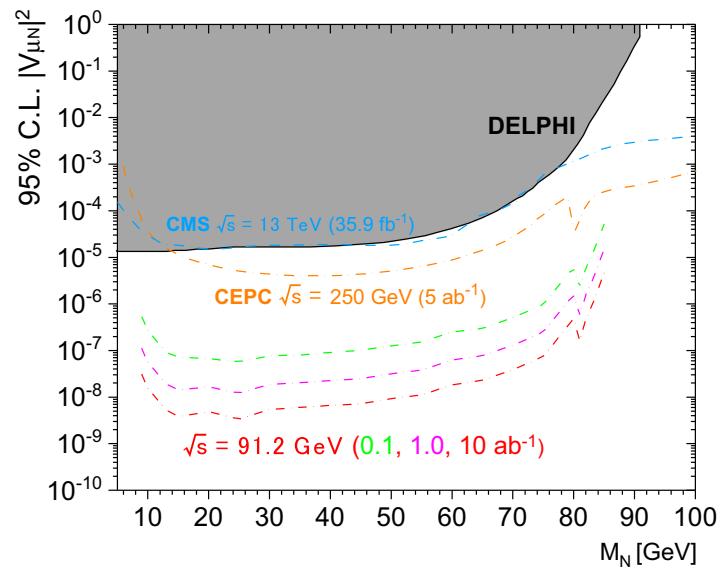

iments [23-25] and the CEPC as a Higgs factory [36]. The green, pink and red curves correspond to integrated luminosities of $0.1 \mathrm{ab}^{-1}$, $1.0 \mathrm{ab}^{-1}$ and $10 \mathrm{ab}^{-1}$, respectively. See text for details

heavy neutrino is most likely to decay before it can fly out of the detector. Comparing $\mathcal{O}\left(10^{-9}\right)$ with the bounds in Fig. 6, one can find that the bounds in the cases with $\mathcal{L}=0.1$ and $1 \mathrm{ab}^{-1}$ are not affected by the limited size of the detector, and that the case with $\mathcal{L}=10 \mathrm{ab}^{-1}$ is also basically safe.

\section{Conclusion}

To conclude, we have presented a study of possible heavy neutrino searches at future $Z$-factories. For different heavy neutrinos with mass ranging from 10 to $85 \mathrm{GeV}$, we have obtained the expected upper bounds on the production cross sections of their discovery processes $e^{+} e^{-} \rightarrow v N \rightarrow v e j j$ and $e^{+} e^{-} \rightarrow v N \rightarrow v \mu j j$ given by $Z$-factory with $\mathcal{L}=$ $0.1,1$ and $10 \mathrm{ab}^{-1}$, respectively. Under the assumption that the interactions between the heavy neutrinos and the SM particles are only induced by the neutrino mixing, the constraints on the cross sections have been translated to the constraints on the corresponding mixing parameters $\left|V_{e N}\right|^{2}$ and $\left|V_{\mu N}\right|^{2}$, which, depending on the luminosity setup, are typically improved by two to four orders compared to the DELPHI constraints [21] and the CMS constraints [22]. We also find that the future $Z$-factories will set much more stringent constraints on $\left|V_{e N}\right|^{2}$ than the $0 \nu 2 \beta$ decay experiments [2325] by one to three orders, and on $\left|V_{\mu N}\right|^{2}$ than the CEPC as a Higgs factory [36] by two to three orders, given the heavy neutrino mass is smaller than $80 \mathrm{GeV}$.

Acknowledgements This work is supported by the Fundamental Research Funds for the Central Universities under the Grant no. lzujbky2019-it08, the National Natural Science Foundation of China under the Grant no. U1732101, the Gansu Natural Science Fund under the Grant no. 18JR3RA265 and the Deutsche Forschungsgemeinschaft (DFG) within research unit FOR 1873 (QFET). We are grateful to Qing-Hong 
Cao, Cheng Chen, Min He, Qiang Li, Qi-Shu Yan and Ye-Ling Zhou for useful discussions. In addition, JND would like to thank Ying Guan for her encouragement.

Data Availability Statement This manuscript has no associated data or the data will not be deposited. [Authors' comment: The data has a too big size to be provided online.]

Open Access This article is distributed under the terms of the Creative Commons Attribution 4.0 International License (http://creativecomm ons.org/licenses/by/4.0/), which permits unrestricted use, distribution, and reproduction in any medium, provided you give appropriate credit to the original author(s) and the source, provide a link to the Creative Commons license, and indicate if changes were made.

Funded by SCOAP ${ }^{3}$.

\section{References}

1. Y.Fukuda et al., Super-Kamiokande Collaboration. Phys. Rev. Lett. 81, 1562 (1998). arXiv:hep-ex/9807003

2. Q.R. Ahmad et al., SNO Collaboration. Phys. Rev. Lett. 89, 011301 (2002). arXiv:nucl-ex/0204008

3. P. Minkowski, Phys. Lett. 67B, 421 (1977)

4. R.N. Mohapatra, G. Senjanovic, Phys. Rev. Lett. 44, 912 (1980)

5. T. Yanagida, Prog. Theor. Phys. 64, 1103 (1980)

6. J. Schechter, J.W.F. Valle, Phys. Rev. D 22, 2227 (1980)

7. M. Magg, C. Wetterich, Phys. Lett. 94B, 61 (1980)

8. T.P. Cheng, L.F. Li, Phys. Rev. D 22, 2860 (1980)

9. G. Lazarides, Q. Shafi, C. Wetterich, Nucl. Phys. B 181, 287 (1981)

10. R.N. Mohapatra, G. Senjanovic, Phys. Rev. D 23, 165 (1981)

11. R. Foot, H. Lew, X.G. He, G.C. Joshi, Z. Phys. C 44, 441 (1989)

12. S.L. Glashow, N.A.T.O. Sci, Ser. B 61, 687 (1980)

13. T. Asaka, M. Shaposhnikov, Phys. Lett. B 620, 17 (2005). arXiv:hep-ph/0505013

14. T. Asaka, S. Blanchet, M. Shaposhnikov, Phys. Lett. B 631, 151 (2005). arXiv:hep-ph/0503065

15. T. Appelquist, R. Shrock, Phys. Lett. B 548, 204 (2002). arXiv:hep-ph/0204141

16. T. Appelquist, M. Piai, R. Shrock, Phys. Rev. D 69, 015002 (2004). arXiv:hep-ph/0308061

17. M. Shaposhnikov, Nucl. Phys. B 763, 49 (2007). arXiv:hep-ph/0605047

18. J. Kersten, A.Y. Smirnov, Phys. Rev. D 76, 073005 (2007). arXiv:0705.3221 [hep-ph]

19. K. Moffat, S. Pascoli, C. Weiland, arXiv:1712.07611 [hep-ph]

20. A. Caputo, P. Hernandez, M. Kekic, J. López-Pavón, J. Salvado, Eur. Phys. J. C 77(4), 258 (2017). arXiv:1611.05000 [hep-ph]

21. P. Abreu et al. DELPHI Collaboration, Z. Phys. C. 74, 57 (1997) Erratum: [Z. Phys. C 75, 580 (1997)]

22. A.M. Sirunyan et al. CMS Collaboration, Phys. Rev. Lett. 120(22), 221801 (2018) https://doi.org/10.1103/PhysRevLett.120.221801. arXiv:1802.02965 [hep-ex]

23. S.R. Elliott, J. Engel, J. Phys. G 30, R183 (2004). arXiv:hep-ph/0405078

24. P. Benes, A. Faessler, F. Simkovic, S. Kovalenko, Phys. Rev. D 71 , 077901 (2005). arXiv:hep-ph/0501295

25. W. Rodejohann, Int. J. Mod. Phys. E 20, 1833 (2011). arXiv:1106.1334 [hep-ph]
26. A. Atre, T. Han, S. Pascoli, B. Zhang, JHEP 0905, 030 (2009). arXiv:0901.3589 [hep-ph]

27. J. Guimaraes da Costa et al., CEPC Study Group, arXiv:1811.10545 [hep-ex]

28. H. Baer et al. arXiv:1306.6352 [hep-ph]

29. M. Bicer et al., TLEP Design Study Working Group. JHEP 1401, 164 (2014). arXiv:1308.6176 [hep-ex]

30. Q. Qin, Q. Li, C.D. Lü, F.S. Yu, Eur. Phys. J. C 78(10), 835 (2018). arXiv:1711.07243 [hep-ph]

31. P.S.B. Dev, R.N. Mohapatra, Y. Zhang, Phys. Rev. Lett. 120(22), 221804 (2018). [arXiv:1711.08430 [hep-ph]]

32. T. Li, M.A. Schmidt, arXiv:1809.07924 [hep-ph]

33. A. Ali, A.Y. Parkhomenko, Q. Qin, W. Wang, Phys. Lett. B 782, 412 (2018). arXiv: 1805.02535 [hep-ph]

34. S. Cheng, Q. Qin, Phys. Rev. D 99(1), 016019 (2019). arXiv: 1810.10524 [hep-ph]

35. W. Liao, X.H. Wu, Phys. Rev. D 97(5), 055005 (2018). arXiv:1710.09266 [hep-ph]

36. Y. Zhang, B. Zhang, JHEP 1902, 175 (2019). arXiv:1805.09520 [hep-ph]

37. F. del Aguila, J.A. Aguilar-Saavedra, Nucl. Phys. B 813, 22 (2009). https://doi.org/10.1016/j.nuclphysb.2008.12.029. arXiv:0808.2468 [hep-ph]

38. A. Das, N. Okada, Phys. Rev. D 88, 113001 (2013). arXiv:1207.3734 [hep-ph]

39. C. Degrande, O. Mattelaer, R. Ruiz, J. Turner, Phys. Rev. D 94(5), 053002 (2016). https://doi.org/10.1103/PhysRevD.94. 053002. arXiv:1602.06957 [hep-ph]

40. A. Das, P. Konar, S. Majhi, JHEP 1606, 019 (2016). arXiv:1604.00608 [hep-ph]

41. A. Abada, N. Bernal, M. Losada, X. Marcano, JHEP 1901, 093 (2019). https://doi.org/10.1007/JHEP01(2019)093. arXiv:1807.10024 [hep-ph]

42. A. Das, S. Jana, S. Mandal, S. Nandi, arXiv:1811.04291 [hep-ph]

43. S. Pascoli, R. Ruiz, C. Weiland, arXiv:1812.08750 [hep-ph]

44. S. Antusch, E. Cazzato, O. Fischer, JHEP 1612, 007 (2016). arXiv:1604.02420 [hep-ph]

45. S. Antusch, E. Cazzato, M. Drewes, O. Fischer, B. Garbrecht, D. Gueter, J. Klaric, JHEP 1809, 124 (2018). arXiv:1710.03744 [hep$\mathrm{ph}]$

46. Y. Chikashige, R.N. Mohapatra, R.D. Peccei, Phys. Lett. 98B, 265 (1981)

47. J. Schechter, J.W.F. Valle, Phys. Rev. D 25, 774 (1982)

48. B.W. Lee, R.E. Shrock, Phys. Rev. D 16, 1444 (1977)

49. J. Alwall, M. Herquet, F. Maltoni, O. Mattelaer, T. Stelzer, JHEP 1106, 128 (2011). arXiv: 1106.0522 [hep-ph]

50. A. Alloul, N.D. Christensen, C. Degrande, C. Duhr, B. Fuks, Comput. Phys. Commun. 185, 2250 (2014). arXiv:1310.1921 [hep-ph]

51. N.D. Christensen, C. Duhr, Comput. Phys. Commun. 180, 1614 (2009). arXiv:0806.4194 [hep-ph]

52. C. Degrande, C. Duhr, B. Fuks, D. Grellscheid, O. Mattelaer, T. Reiter, Comput. Phys. Commun. 183, 1201 (2012). arXiv:1108.2040 [hep-ph]

53. T. Sjöstrand et al., Comput. Phys. Commun. 191, 159 (2015). arXiv: 1410.3012 [hep-ph]

54. J. de Favereau et al., DELPHES 3 Collaboration. JHEP 1402, 057 (2014). arXiv:1307.6346 [hep-ex]

55. M. Tanabashi et al.,. Particle Data Group, Phys. Rev. D. 98(3), 030001 (2018) 